\title{
Beyond Psychosis Risk: Early Clinical Phenotypes in Mental Disorder and the Subthreshold Pathway to Safe, Timely and Effective Care
}

\author{
Patrick D. McGorry \\ Orygen Youth Health Research Centre, Centre for Youth Mental Health, University of Melbourne, \\ Melbourne, Vic., Australia
}

The past two decades have witnessed a genuine paradigm shift, which has enabled the earliest clinical phenotypes of psychotic illness to be defined, and for the prediction and indicated prevention of fully-fledged psychotic disorder to become a realistic possibility [1]. This development has not been without controversy. Ambivalence, within not only the general public but also the mental health professions, concerning the safety and value of mental health care as currently delivered has meant that moving to treat mental disorders in their earliest clinical stages has led to intense debate. This debate, while distorted at times by ideological forces and vested interests, is nevertheless a healthy and necessary one. However, it has wider implications for early intervention beyond psychosis.

The key and potent concept here is indicated prevention, where subthreshold symptoms, buttressed where possible with key biomarkers, can be used not only to define a need for care in their own right but also a substantial but not inevitable risk of more serious disorder [2]. This is different from selective prevention, where asymptomatic patients, such as those with elevated fasting blood glucose and 'prediabetes', are at elevated risk of future fully-fledged illness. In defining subthreshold mental states that justify a need for care, we need to consider the complex issue of the boundary with the rather fuzzy no-

\section{KARGER}

E-Mail karger@karger.com

www.karger.com/psp tion of 'normality'. It is challenging to rigidly define the boundary between this 'normality' and the earliest clinical phenotypes of mental disorder with a need for care. A key question is how critical or feasible it is to create such a precise definition.

Both contributors to the current debate in Psychopathology agree that the ultra-high risk concept has been a genuine advance, one which justifies its inclusion in the DSM-5 at least as a condition requiring further study. In my view, Carpenter [3] has dealt effectively with most of the arguments against its full inclusion, which generally relate to the poor quality and resourcing of mental health care rather than the validity of the approach. We know, as Nelson [4] points out, that the universal neglect of mental health care, and particularly the failure to fund psychosocial care, will often lead to premature treatment with medications as well as potentially enhancing stigma through late intervention in tertiary settings. However, the latter, as we know from our experience in Australia, are problems that can be solved [5]. Furthermore, these genuine fears of overtreatment and harm need to be balanced against the current reality of undertreatment, which is the norm. The case for full inclusion, however, was probably undermined not so much by the poor reliability found in the flawed field trial, but by the reducing
(C) 2014 S. Karger AG, Basel

0254-4962/14/0475-0285\$39.50/0 
predictive power of the criteria over time, which have been recognised to be dependent on the population studied and the level of intrinsic risk. This issue may not have been properly acknowledged by some advocates for inclusion. The other factor that undermined the case is that while the ultra-high risk criteria have a stronger valence for subsequent psychosis, they also capture markedly elevated risk for mood, anxiety and substance use disorders and deliberate self-harm [1]. They have only partial specificity. The key value of the ultra-high risk concept is therefore prototypical; there is a much bigger fish to fry, namely, the wider benefit of capturing early clinical phenotypes, which are cross-diagnostic with respect to traditional diagnostic concepts, themselves late-stage concepts derived from late 19th and early 20th century tertiary care settings. All major psychiatric disorders have early clinical stages or 'prodromes' during which sustained distress and disability embed and care is needed, yet well before traditional diagnostic 'clarity' is achieved [6]. Introducing one earlier clinical phenotype (the attenuated psychosis syndrome) within a single diagnostic silo in a relatively unchanged DSM-5 (notwithstanding a degree of specificity) without reference to other diagnostic streams would produce an asymmetry which could obscure the wider issue and undermine wider reform.

The clinical staging model adapted from general medicine ensures that interventions are proportional to both need and the risk of 'extension' of the clinical phenotype and its consequences. It recognises that persistent and multiple 'microphenotypes' of disturbance can justify a need for care on their immediate merits as well as risk for progression to more familiar 'macrophenotoypes'; however, it recognises the need for blending dimensional and categorical models. Staging moves outside the current diagnostic silos to include the full spectrum of disorder, and while highly congruent with notions of an extended phenotype for individual disorders, which involves continuity with the healthy population, it places strong diagnostic emphasis on where a person sits in the evolution of the clinical phenotype. This is with the goal of improving diagnostic utility in relation to treatment selection and evaluation. Staging also has a longer-term heuristic goal of facilitating linkages between biological and cognitive markers and clinical phenotypes agnostically rather than diagnostically [7].

One might argue that we should allow no more specificity in the diagnostic term or label than is necessary to guide treatment selection. We might start to see some of this specificity emerge with the advent of clearer, more stable and sustained syndromes or of severe syndromes or macrophenotypes such as psychosis, mania, depression, anxiety, addiction and the borderline syndrome, either alone or, more typically, in 'comorbid' blends. In any event, we need a heuristic strategy, which clinical staging provides, and I hope this can assemble sufficient data to support its inclusion somehow in the next editions of the DSM and ICD. The attenuated psychosis syndrome remains an important stalking horse for that next step in redeeming psychiatric diagnosis and improving its utility, safety and validity [8].

\section{References}

$\checkmark 1$ Fusar-Poli P, Borgwardt S, Bechdolf A, Addington J, Riecher-Rössler A, Schultze-Lutter F, et al: The psychosis high-risk state: a comprehensive state-of-the-art review. JAMA Psychiatry 2013;70:107-120.

2 McGorry PD: Risk syndromes, clinical staging and DSM V: new diagnostic infrastructure for early intervention in psychiatry. Schizophr Res 2010;120:49-53.

-3 Carpenter WT: Attenuated psychosis syndrome: need for debate on a new disorder. Psychopathology DOI: 10.1159/000365221.
4 Nelson B: Attenuated psychosis syndrome: don't jump the gun. Psychopathology DOI: $10.1159 / 000365291$.

5 McGorry P, Bates T, Birchwood M: Designing youth mental health services for the 21st century: examples from Australia, Ireland and the UK. Br J Psychiatry 2013;54:S30-S35.

6 McGorry P: Early clinical phenotypes and risk for serious mental disorders in young people: need for care precedes traditional diagnoses in mood and psychotic disorders. Can J Psychiatry 2013;58:19-21.
7 McGorry PD: The next stage for diagnosis: validity through utility. World Psychiatry 2013;12:213-215.

8 McGorry P, van Os J: Redeeming diagnosis in psychiatry: timing versus specificity. Lancet 2013;381:343-345. 